\title{
Sentinel lymph node biopsy in medullary thyroid microcarcinomas
}

\author{
Nada Santrac ${ }^{1)}$, Ivan Markovic ${ }^{1), 2)}$, Natasa Medic Milijic ${ }^{3)}$, Merima Goran $^{1), 2)}$, Marko Buta ${ }^{1,2),}$ \\ Igor Djurisic ${ }^{1)}$ and Radan Dzodic ${ }^{1), 2)}$ \\ 1) Surgical Oncology Clinic, Institute for Oncology and Radiology of Serbia, Belgrade, 11000, Serbia \\ 2) School of Medicine, University of Belgrade, Belgrade, 11000, Serbia \\ 3) Department of Pathology, Institute for Oncology and Radiology of Serbia, Belgrade, 11000, Serbia
}

\begin{abstract}
The aim of this prospective study was to analyze accuracy of sentinel lymph node biopsy with methylene blue dye for intraoperative detection of lateral metastases in clinically NOM0 medullary microcarcinomas with calcitonin $<1,000 \mathrm{pg} / \mathrm{mL}$ and selection of true-positive patients for one-time therapeutic lateral dissection. In addition to total thyroidectomy and central neck dissection, all patients had bilateral sentinel biopsy of jugulo-carotid regions after methylene blue injection to decide upon necessity for lateral dissection. If sentinels were benign on frozen section, additional nonsentinels were extirpated, with no further lateral dissection. If sentinels were malignant, one-time lateral dissection was performed. 20 patients were included in this study. Hereditary disease form was observed in $3 / 20(15 \%)$ of patients with RET proto-oncogene mutation C634F; remaining 17/20 (85\%) were negative for germline mutations. There were no allergic reactions to methylene blue and identification rate of sentinels was $100 \%$. In total, $2 / 20(10 \%) \mathrm{cN} 0$ patients had lymphonodal metastases, thus were reclassified as pN1b. Remaining 18/20 (90\%) were classified pN0 based on standard pathohistology. Frozen section findings on sentinels were $100 \%$ match with standard pathohistology, and there were no skip metastases in lateral compartments. Sensitivity, specificity and accuracy of sentinel biopsy method with methylene dye and frozen section were $100 \%$. Dzodic's sentinel lymph node biopsy method can be used for intraoperative assessment of lateral compartments and optimization of initial surgery of medullary microcarcinomas with calcitonin $<1,000 \mathrm{pg} / \mathrm{mL}$. This way, cN0 patients with sentinel metastases can receive one-time lateral dissection, and those without benefit from less extensive surgery.
\end{abstract}

Key words: Medullary thyroid microcarcinoma, Calcitonin below 1,000, Methylene blue dye, Sentinel lymph node biopsy, Lateral neck dissection

\begin{abstract}
MEDULLARY THYROID CARCINOMA (MTC) is a rare thyroid malignancy of $\mathrm{C}$ cell origin, characterized by the secretion of a peptide hormone calcitonin (CT). It is accounting for $3 \%$ (adults) to $10 \%$ (children) of thyroid cancers [1]. MTC appears in two forms: sporadic $(75 \%)$ and hereditary $(25 \%)$, with similar gender distribution [1]. MTCs have more aggressive behavior than differentiated thyroid carcinomas, and tend to spread relatively early into regional lymph nodes (LN) [2]. It has been reported that $80 \%$ of patients with palpable MTCs have central compartment LN metastases at the time of diagnosis, while $75 \%$ and $47 \%$ (respectively) have $\mathrm{LN}$ metastases in ipsilateral and contralateral jugulo-carotid regions [3].
\end{abstract}

Submitted Sep. 21, 2019; Accepted Oct. 31, 2019 as EJ19-0409 Released online in J-STAGE as advance publication Dec. 3, 2019 Correspondence to: Nada Santrac, $\mathrm{MD}, \mathrm{PhD}(\mathrm{c})$, Surgical Oncology Clinic, Institute for Oncology and Radiology of Serbia, Pasterova 14, Belgrade 11000, Serbia.

E-mail: santrac.nada@gmail.com
Medullary thyroid microcarcinomas (micro-MTC) are defined as tumors with diameter $10 \mathrm{~mm}$ or less [4]. Although clinical relevance of sporadic and hereditary micro-MTCs is debatable in literature, there is a significant disease burden associated with this entity [5-11].

Serum CT level is a precise MTC marker, both for preoperative diagnostics, as well as for postoperative assessment of disease relapse. It usually correlates with tumor size and disease extent. However, LN and distant metastases are diagnosed, as well, in patients with lower preoperative CT values [12].

Surgery is the only curative treatment for MTCs. Aim of initial surgery is adequate tumor and LN clearance to achieve good loco-regional disease control [1-3, 13], and, if possible, biochemical cure, commonly used as a surrogate marker for surgical cure [10]. False negative findings on preoperative physical examination and ultrasonography of regional LNs can lead to initial undertreatment of patients with this disease. Due to permanent concerns regarding under- and over-treatment of patients, 
surgical recommendations for MTC management are not unanimous, but majority of those agree upon necessity of total thyroidectomy (TT) with prophylactic central lymph node dissection (CLND) [1, 2, 13-18]. The necessity of lateral lymph node dissections (LLND) in clinically evident disease is indisputable; however, its extent in clinically $\mathrm{N} 0$ patients is a matter of debate, ranging in the literature from (a) bilateral prophylactic LLND [10, $12,16,19]$, as the most radical approach; or (b) sampling of lateral LNs, as less radical strategy to determine the necessity of complete LLND in histologically proven metastatic disease [20]; to (c) performing ipsilateral LLND only in case of positive central LNs [1, 21-23].

In the context of timely detection and treatment of subclinical, occult, LN disease, while, on the other hand, not over-treating patients without metastases, some authors perform sentinel lymph node biopsy (SLNB), first introduced by Kelemen and co-authors in 1998 [24]. Based on literature data, this method is most commonly used in selection of patients for CLND in differentiated thyroid carcinomas [25-31]. Given that CLND is performed in our center as a standard surgical protocol for thyroid carcinomas, our leading surgeon Prof. Radan Dzodic (last author) introduced SLNB technique for intraoperative staging of lateral LNs in clinically negative (cN0) patients, and published first results in 2006 [32]. Initially, this method was used for differentiated thyroid carcinomas only, but later for MTCs, as well.

The aim of this paper was to present results of the first study that analyzed accuracy of SLNB with methylene blue dye (MBD) for intraoperative staging of lateral LNs in cN0 micro-MTCs with serum CT levels below 1,000 $\mathrm{pg} / \mathrm{mL}$, and its usefulness in selection of patients for onetime therapeutic LLND.

\section{Materials and Methods}

\section{Patients}

This prospective study started in 2007, as a part of the project of the Ministry of Science of Republic of Serbia (project number: 111601/2005; project name: "Early detection of lymphonodal metastases by pathohistological verification of sentinel lymph nodes in malignant epithelial tumors"), with aim to include patients with suspicious or confirmed sporadic or hereditary MTCs meeting following criteria: (a) tumor diameter $10 \mathrm{~mm}$ or less, without extrathyroid extension (b) serum CT levels lower than $1,000 \mathrm{pg} / \mathrm{mL}$, (c) clinically negative regional LNs and (d) absence of distant metastases (cM0). It is set in the Institute for Oncology and Radiology of Serbia (IORS).

Patients were either diagnosed primarily in IORS, or they were referred to our center for surgery of suspicious
MTC. MTC diagnosis was set based on (a) presence of specific germline mutations or (b) suspicious thyroid tumor or thyroid goiter on physical examination and neck ultrasonography (US) with elevation of basal serum $\mathrm{CT}$ levels. If basal CT elevation was inconclusive for MTC diagnosis, calcium-stimulating test was performed for evaluation of stimulated $\mathrm{CT}$ increase and decision upon surgery.

Standard preparation for surgery in general anesthesia included: complete thyroid and parathyroid function, complete blood and biochemistry tests, indirect laryngoscopy, chest and tracheal X-ray, abdominal US, internist and anesthesiologist evaluation. In all patients concomitant pheochromocytoma and primary hyperparathyroidism were preoperatively excluded. Preoperative diagnostics was case-oriented in matters of contrastenhanced computerized tomography (CECT) and fine needle aspiration biopsy (FNAB).

Multidisciplinary IORS Thyroid team decided upon the surgical treatment. Patients were explained in detail the surgical procedure, possible complications and treatment outcomes. Written consent for surgical treatment was obtained from each patient. Surgical protocols for clinically N0 thyroid carcinomas in our center include SLNB with MBD for intraoperative LN staging, and they were approved by IORS Ethical Committee.

\section{Study design}

Gender-, age-, disease- and treatment-specific characteristics of the group were analyzed. Given the defined aim, to assess the accuracy of SLNB for intraoperative staging of lateral LNs, we have compared findings of frozen section analysis (FSA) and standard pathohistological analysis (sPH) on sentinel-LNs, as well as evaluated additional lateral LNs on $\mathrm{sPH}$ for skip metastases (metastases in non-sentinel-LNs). Sensitivity, specificity, positive and negative predictive values and overall accuracy of the method were calculated.

\section{Surgical treatment}

Surgical treatment was uniform for all selected patients, and consisted of TT, prophylactic CLND (level VI), SLNB of both jugulo-carotid regions and FSA of sentinel-LNs. If sentinel-LNs were benign, additional surrounding non-sentinel-LNs from belonging jugulocarotid region were removed for $\mathrm{sPH}$. Whenever metastases were detected in sentinel-LNs on FSA, a therapeutic LLND was done.

Standard Kocher's collar incision is made in the natural skin fold, between anterior borders of sternocleidomastoid muscles. After transection of platysma, middleline dissection and lateralization of infrahyoid muscles, thyroid gland is exposed. Specific surgical technique is 
explained in details in next few steps.

Step 1: Peritumoral thyroid injection of $0.2-0.5 \mathrm{~mL}$ of $1 \%$-MBD solution (using a 27-gauge needle) beneath thyroid capsule, with thermal coagulation of the injection site through surgical tweezers (Fig. 1). In order to avoid MBD leakage, thyroid capsule should not be damaged. Coloring of lymphatic vessels and LNs around thyroid gland is observed immediately (Fig. 2).

Step 2: Removal of the lobe with the suspicious tumor and FSA for pathohistological confirmation of the MTC. Parathyroid glands (PTGs) are not colored with MBD, so their identification and preservation is easier (Fig. 3).

Step 3: Exploration of ipsilateral jugulo-carotid region, focusing on levels II and III, in order to identify

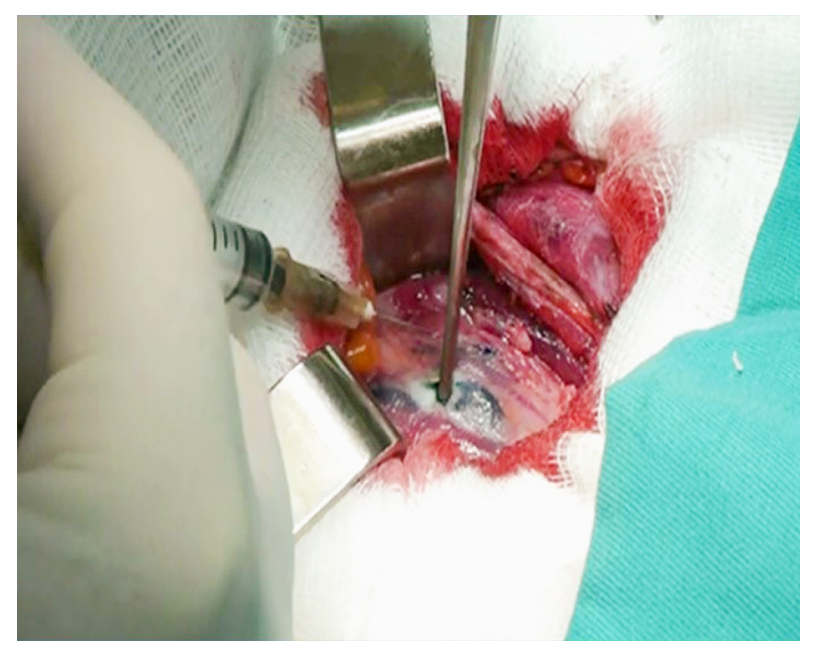

Fig. 1 Injection of $0.2-0.5 \mathrm{~mL}$ of $1 \%$-methylene blue dye solution using a 27-gauge needle in thyroid lobe, beneath thyroid capsule, with thermal coagulation of the injection site through surgical tweezers, to avoid the leakage of the tracer

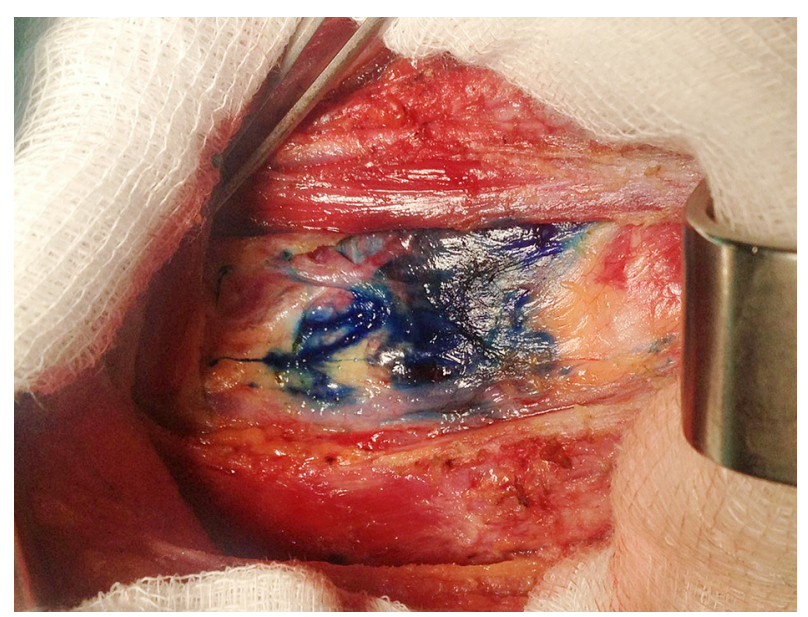

Fig. 2 Methylene blue dye affecting thyroid lobes, lymphatic vessels and lymph nodes of central compartment colored afferent lymphatic vessels (Fig. 4) and bluestained, sentinel-LNs that are meticulously removed (Fig. 5) and examined by FSA. If there are no colored LNs, LN belonging to the colored afferent lymphatic is considered to be the sentinel-LN, thus is removed for FSA.

Step 4: Removal of additional surrounding nonsentinel-LNs if sentinel-LNs are benign on FSA; or onetime therapeutic LLND of the side where sentinel-LN is proven to be malignant on FSA.

Step 5: Completion thyroidectomy and level VI clearance, with careful preservation of PTGs on venousarterial vascular pedicles [33], as well as identification and preservation of both recurrent laryngeal nerves. Due to MBD injection, central LNs are clearly marked with blue, while PTGs remain non-colored, making CLND easier and safer, especially in less experienced hands. If

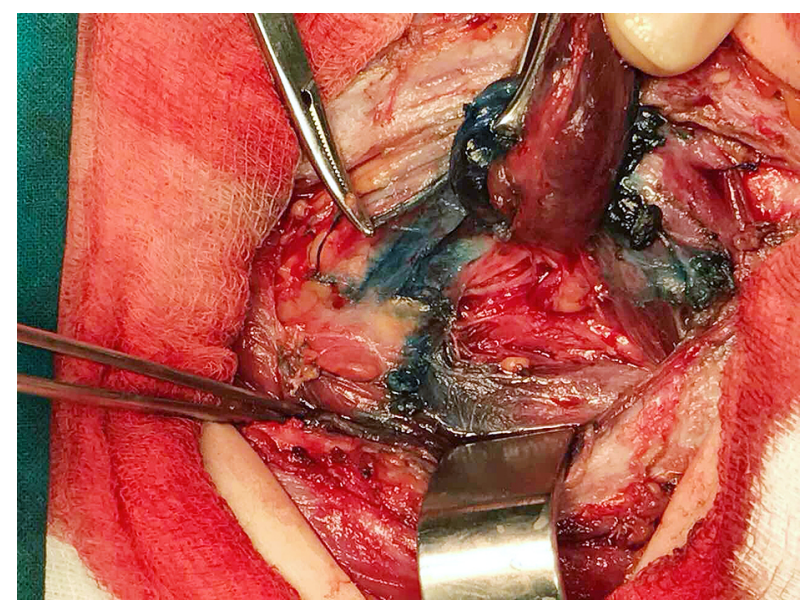

Fig. 3 Removal of thyroid lobe, with preservation of parathyroid glands that are not colored with methylene blue dye, while central lymph nodes uptake the vital dye

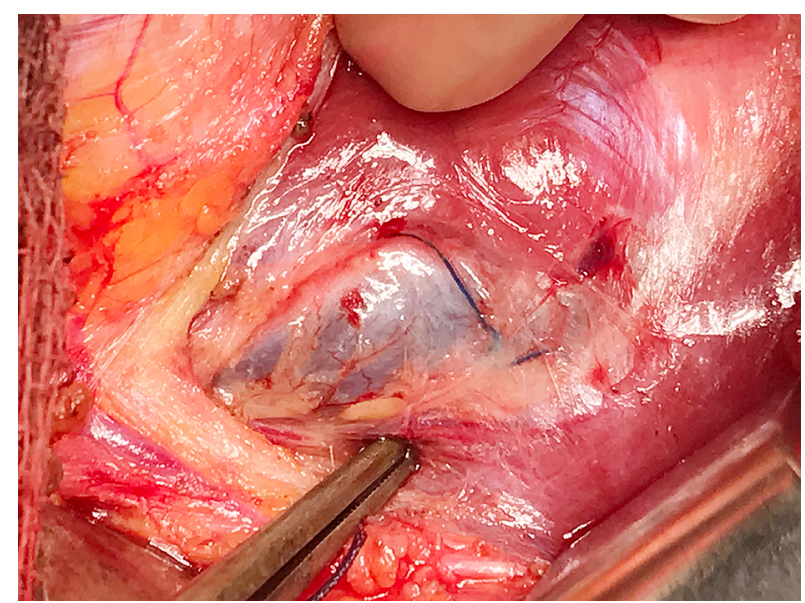

Fig. 4 Identification of blue-stained afferent lymphatic vessel in the jugulo-carotid region 


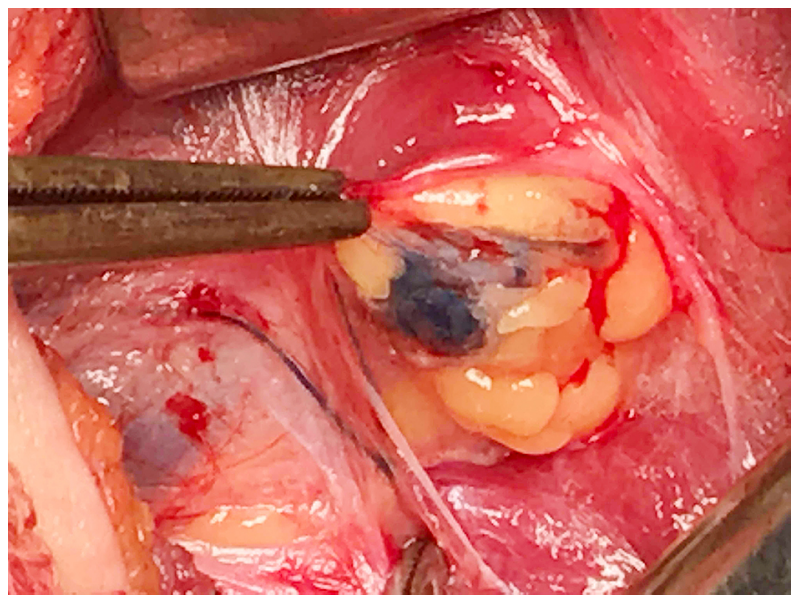

Fig. 5 Identification and extirpation of blue-stained, sentinel, lymph nodes from jugulo-carotid region

a PTG is accidentally removed during CLND or devascularized, it should be minced and autotransplanted by Wells technique into the sternocleidomastoid muscle [34].

Step 6: SLNB of contralateral jugulo-carotid region with FSA and further procedure, as described in step 4.

\section{Postoperative course}

In the postoperative course, all patients were monitored and managed according to our IORS protocols for post-thyroidectomy patients: measurement of parathyroid hormone, serum calcium and phosphorus levels immediately after surgery and on the first postoperative day, and active evaluation of signs/symptoms of hypocalcaemia, laryngeal nerves injuries or other complications.

After postoperative multidisciplinary team evaluation of each patient, L-thyroxin was initiated. General recommendations for follow-up of MTC patients in our center include: serum $\mathrm{CT}$ and CEA measurements to verify biochemical status of the disease, US in all patients, and CECT or ${ }^{99 \mathrm{~m}} \mathrm{Tc}-\mathrm{V}-\mathrm{DMSA}$ scans, if indicated, for local or distant relapse assessment. All patients were followed-up in our tertiary center.

\section{Statistical analysis}

SPSS (SPSS Inc., IBM, Chicago, USA), version 23, was used for statistical analysis. Descriptive statistical methods (frequencies, percentages, mean, standard deviation (SD) and range) were used to summarize data on patients, disease, treatment and outcomes. MedCalc statistical software for Windows was used for evaluation of diagnostic value of the SLNB method (sensitivity, specificity, positive and negative predictive values and overall accuracy).

\section{Results}

In total, from 2007 to 2019, 20 patients met all four criteria and were eligible for inclusion in this study. There was a female predominance in the sample $(90 \%$, $18 / 20$ patients). Mean age of patients was $53.90 \pm 17.10$ (range: 18 to 83 ) years.

\section{Preoperative findings}

Basal CT ranged from 7.2 to $697.0 \mathrm{pg} / \mathrm{mL}$ in the group, with a mean value of $234.5 \mathrm{pg} / \mathrm{mL}$ and great variations among patients $(\mathrm{SD}=217.6)$.

Hereditary form of disease was observed in three patients (15\%) with RET proto-oncogene mutation C634F. Patient 1 was a male, aged 46, with basal CT of $221.6 \mathrm{pg} / \mathrm{mL}$, a suspicious tumor in the left lobe and negative LNs on US and physical examination. Patient 2 was a female, aged 18 , with basal CT of $42.0 \mathrm{pg} / \mathrm{mL}$, a suspicious tumor in the right lobe and clinically negative LNs. Patient 3 was a female, aged 72, with basal CT of $42.6 \mathrm{pg} / \mathrm{mL}$, bilateral thyroid tumors on US and physical examination, and negative LNs. Patient 1 and 2 had no clinical or biochemical signs of adrenal or PTG tumors. Patient 3 had prior surgery for pheochromocytoma and PTG adenoma. Remaining 17 patients (85\%) were negative for germline mutations based on standard genetic testing.

FNAB was done by endocrinologist in 4/20 (20\%) patients, suggesting of colloid goiter in half and normal thyroid tissue in other $50 \%$ of patients.

\section{Surgical treatment}

All patients had TT, prophylactic CLND (level VI), bilateral SLNB of jugulo-carotid regions and FSA of sentinel-LNs. None of the patients had allergic reactions to MBD. In all patients blue-stained LNs were identified in lateral compartments ( $100 \%$ identification rate).

In $90 \%(18 / 20)$ of patients examined sentinel-LNs were benign on FSA (Table 1). Here, additional surrounding non-sentinel-LNs were removed from the respective jugulo-carotid regions for sPH analysis. In $10 \%(2 / 20)$ of patients, both with hereditary MTC (patient 1 and 2), LN metastases were detected in sentinel-LNs on FSA, thus therapeutic LLNDs of respective jugulo-carotid compartments were done. Patient 1 had bilateral LLND of regions II-V, since sentinel-LN metastases were found in both jugulo-carotid regions, while LLND was performed on the right side in patient 2.

\section{Pathohistological reports}

Unilateral unifocal tumors were found in $90 \%(18 / 20)$ of patients, presenting as sporadic MTC in 16 and hereditary form in 2 patients. One patient (5\%) with sporadic disease form had unilateral multifocal MTC, 
Table 1 Pathohistological characteristics of sentinel lymph nodes

\begin{tabular}{lc}
\hline SLN characteristics & $\%(n)$ of patients \\
\hline Identification rate & $100 \%(20 / 20)$ \\
FSA findings & \\
$\quad$ benign & $90 \%(18 / 20)$ \\
$\quad$ malignant & $10 \%(2 / 20)$ \\
sPH findings & \\
$\quad$ benign & $90 \%(18 / 20)$ \\
$\quad$ malignant & $10 \%(2 / 20)$ \\
FSA and sPH match & $100 \%(20 / 20)$ \\
\hline TOTAL & $100 \%(20)$ \\
\hline
\end{tabular}

Legend: SLN, sentinel lymph node; FSA, frozen section analysis; sPH, standard pathohistology

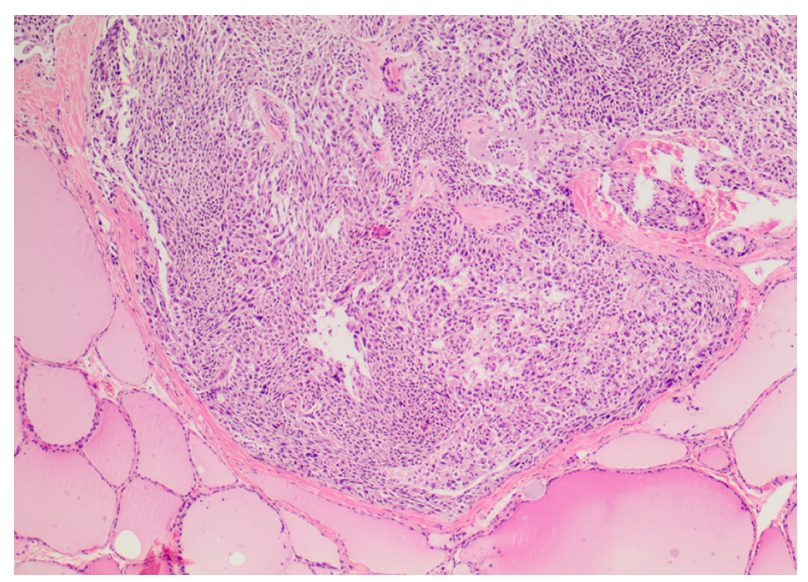

Fig. 6 Medullary thyroid carcinoma in a patient with C634F mutation and bilateral tumors (haematoxylin-eosin stain; magnification: $40 \times$ )

and one patient (5\%) with hereditary MTC had bilateral microcarcinomas (Fig. 6). As shown in Table 2, mean tumor size in the group was $8.05 \pm 2.04$ (range: 3 to 10 ) $\mathrm{mm}$. Capsular and lymphovascular invasion was not observed in this group. Associated thyroid disease was present in $50 \%(10 / 20)$ of patients, which is given in details in Table 3.

The average number of examined sentinel-LNs was $3.10 \pm 1.48$ (range: $2-7$ ), and total number of sentinelLN metastases in two patients selected on FSA was 1/1 LN per both lateral compartments in patient 1 (Fig. 7) and 1/4 LNs in ipsilateral compartment of patient 2 .

A mean of $11.15 \pm 6.33$ (range: 6-28) non-sentinelLNs were removed from jugulo-carotid regions (LLND included). In 2 patients that were FSA-selected for onetime LLND, number of additional lateral LN metastases was $2 / 11$ and $2 / 12$ in bilateral LLND in patient 1 , and $1 / 15$ in unilateral LLND in patient 2.
Table 2 Pathohistological characteristics of medullary thyroid microcarcinomas

\begin{tabular}{lc}
\hline Tumor characteristics & $\%(n)$ of patients \\
\hline Tumor size & $8.05 \pm 2.04 \mathrm{~mm}$ \\
average & $3-10 \mathrm{~mm}$ \\
range & \\
Capsular invasion & $100 \%(20 / 20)$ \\
no & $0 \%(0 / 20)$ \\
yes & \\
Lymphovascular invasion & $100 \%(20 / 20)$ \\
no & $0 \%(0 / 20)$ \\
yes & \\
Tumor pattern & $90 \%(18 / 20)$ \\
unilateral unifocal & $88.9 \%(16 / 18)$ \\
sporadic & $11.1 \%(2 / 18)$ \\
hereditary & $5 \%(1 / 20)$ \\
unilateral multifocal* & $5 \%(1 / 20)$ \\
\hline bilateral, multicentric** & $100 \%(20)$ \\
\hline TOTAL &
\end{tabular}

Legend: * sporadic MTC; ** hereditary MTC

Table 3 Pathohistological reports on associated thyroid diseases

\begin{tabular}{lc}
\hline Associated thyroid disease & $\%(n)$ of patients \\
\hline None & $50 \%(10 / 20)$ \\
\hline Hashimoto's thyroiditis & $15 \%(3 / 20)$ \\
Follicular adenoma & $15 \%(3 / 20)$ \\
C-cell hyperplasia & $5 \%(1 / 20)$ \\
Micro-PTC & $10 \%(2 / 20)$ \\
$\begin{array}{l}\text { Micro-PTC + Hurthle cell tumor of uncertain } \\
\text { malignant potential }\end{array}$ & $5 \%(1 / 20)$ \\
\hline TOTAL & $100 \%(20)$ \\
\hline
\end{tabular}

Legend: PTC, papillary thyroid carcinoma

Average number of dissected central LNs was 9.61 \pm 2.54 (range: $5-15$ ). Central LN metastases were verified in those 2 patients with sentinel-LN metastases on FSA. In patient 1, 3/12 central LNs were affected, while patient 2 had metastasis in 1/9 dissected central LNs.

\section{SLNB diagnostic value}

Definite pathohistological reports on sentinel-LNs were $100 \%$ match with FSA findings.

In total, $10 \%(2 / 20)$ of patients were reclassified from cN0 to pN1b due to SLNB with MBD and FSA. Remaining $90 \%(18 / 20)$ of patients were confirmed to be pN0 based on sPH analysis of central LNs, sentinel-LNs and non-sentinel-LNs of lateral compartments. 


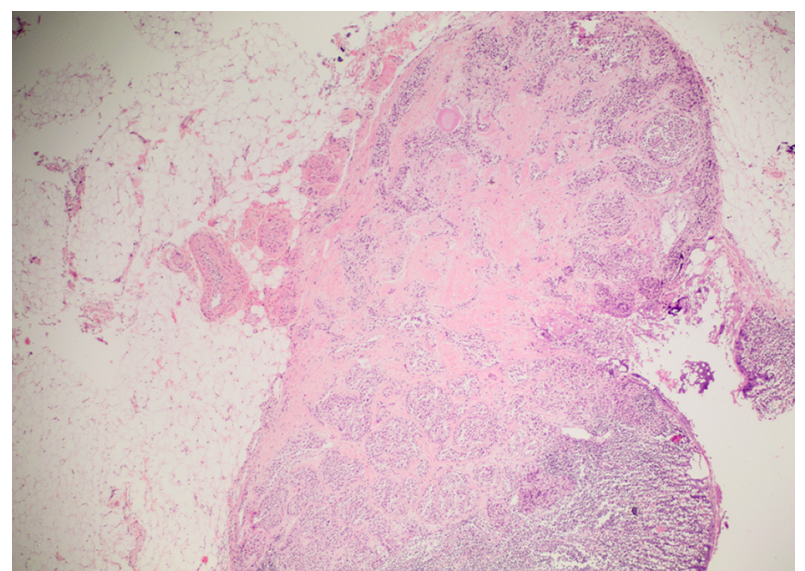

Fig. 7 Sentinel lymph node metastasis in a patient with C634F mutation and bilateral tumors (haematoxylin-eosin stain; magnification: $20 \times$ )

In 18 patients that had negative sentinel-LNs on FSA and $\mathrm{SPH}$ there were no skip metastases in additional LNs that were extirpated for analysis. In 2 patients that were FSA-selected for one-time LLND of affected compartments, sPH examination revealed additional LN metastases in dissected compartments, meaning positive sentinel-LNs on FSA were $100 \%$ predictive for presence of additional lateral LN metastases.

Sensitivity, specificity, positive and negative predictive value of SLNB method with MBD and FSA were $100 \%$. The overall accuracy of the method was $100 \%$ (Table 4).

\section{Postoperative follow-up data}

In the immediate postoperative course, all patients were carefully evaluated for complications. There were neither postoperative bleedings, nor permanent hypoparathyroidism in the group. One patient (5\%) was with mild hoarseness, that resolved spontaneously (thus was attributed to neuropraxia), but he also had a prolonged lymphatic leakage that could not have been resolved with conservative approach, so a re-do surgery was performed with suture of injured lymphatic vessel on the right side and full recovery after being discharged from hospital.

Patients were followed-up in average $79.75 \pm 36.40$ (range: 1-145.75) months. During this time, serum CT and CEA were within normal ranges in all patients, suggesting complete biochemical cure.

\section{Discussion}

Although micro-MTCs have earlier been considered harmless, studies that analyzed clinical behavior of this entity have shown a significant disease burden associated with micro-MTCs [5-11].

Role of CT screening for active detection of MTCs, especially in nodular goiter patients, remains debatable in the literature $[10,19,35]$; however, this is the routine clinical practice in our center.

Every increase in basal CT levels should raise suspicion of MTC and further diagnostics should be performed. Still, lower CT values might not always be of diagnostic significance, due to potential confounding effects of comorbidities (e.g. sporadic C-cell hyperplasia, hypercalcaemia, neuroendocrine tumors, etc.), drugs (e.g. proton pump inhibitors), smoking, or due to assay related factors, gender specific differences, and other [1]. Stimulated CT should help in differential diagnosis, although test is not available in all countries.

Preoperative CT usually correlates to tumor size and disease extent; however, it can lead to inadequate disease staging and under-treatment, given that patients may have LN metastases in lower CT levels [12]. In our study, two patients with LN metastases had basal CT levels of 221.6 and $42.0 \mathrm{pg} / \mathrm{mL}$, while those with higher values, over $500 \mathrm{pg} / \mathrm{mL}$, for example, were staged pN0. Physical examination and US evaluation are used to guide the extent of LN surgery, but are not always reliable $[1,15]$. Intraoperative assessment of LNs by a surgeon was also shown not to be accurate enough, with sensitivity of $64 \%$ [36].

General recommendation for treatment of MTC is TT with prophylactic CLND, even in patients with microMTCs [1, 2, 13-18], and this surgical strategy is used in

Table 4 Diagnostic values of sentinel lymph node biopsy method using methylene blue dye and frozen section analysis

\begin{tabular}{|c|c|c|c|c|}
\hline \multirow{2}{*}{ SLN status } & \multicolumn{2}{|c|}{ Lateral lymph nodes status } & \multirow{2}{*}{ TOTAL $(n)$} & \multirow{2}{*}{ Diagnostic SLN biopsy value } \\
\hline & Positive $(n)$ & Negative $(n)$ & & \\
\hline Positive $(n)$ & 2 & 0 & 2 & $\mathrm{PPV}=100 \%(2 / 2)$ \\
\hline Negative $(n)$ & 0 & 18 & 18 & $\mathrm{NPV}=100 \%(18 / 18)$ \\
\hline \multirow[t]{2}{*}{ TOTAL } & 2 & 18 & 20 & \\
\hline & $\mathrm{SN}=100 \%(2 / 2)$ & $\mathrm{SP}=100 \%(18 / 18)$ & $\mathrm{FNR}=0 \%(0 / 2)$ & $\mathrm{ACC}=100 \%$ \\
\hline
\end{tabular}

Legend: $n$, number of patients; SLN, sentinel lymph node; SN, sensitivity; SP, specificity; FNR, false negative rate; PPV, positive predictive value; NPV, negative predictive value; ACC, accuracy 
our center, as well. However, the necessity and the extent of LLND are debatable in the referent literature. Undoubtedly, in clinically evident lateral LN metastases (macroscopically or by US), therapeutical LLND is required. However, recommendations are not unanimous regarding the indications for LLND in the clinical absence of metastases.

Many studies upon clinical behavior of MTCs acknowledged that LN metastases occur early in the course of MTC, regardless the tumor size [2, 37]. For example, at the time of diagnosis, over $70 \%$ of patients with palpable MTC [36] and over 35\% of micro-MTCs [9] have LN metastases. In addition, the risk of lateral $\mathrm{LN}$ involvement in patients with central $\mathrm{LN}$ metastases is at least $70 \%$ [23], while over a third of patients with central also have contralateral lateral LN metastases [38].

Due to these data reports on high rate of LN metastases in MTCs and many reports on prognostic significance of initial surgery for patients' survival [39], significant number of surgeons have approached to MTC treatment more extensively, even in the absence of lateral LN involvement. Namely, majority of surgical teams [2, $10,12,16,19,36,37]$ advocate bilateral prophylactic LLND in all MTC patients. The aim is to achieve higher biochemical cure rate (postoperative CT normalization), lower rate of reoperations and better survival rates. Some data suggest that contralateral LLND can be omitted in sporadic MTCs, if there is no evidence of central and ipsilateral lateral LN involvement, but it is still necessary to perform routine bilateral LLNDs in hereditary forms $[1,2]$.

Less radical surgical approach for clinically N0 patients implies performing ipsilateral LLND only in case of positive central LNs [1, 21-23], or if sampling of lateral LNs harbor metastatic disease [20].

Break-through in minimally invasive approach to LN management in thyroid carcinomas has to be contributed to Kelemen [24], who used isosulfan blue dye (IBD) to mark LNs of the central and lateral neck regions, based on well-established, widely used model of SLNB in breast carcinoma and melanoma. Main goals of SLNB are: to accurately detect LN metastases, to optimize the extent of LN surgery (patients with metastases are selected for complete dissection, others not), to minimize morbidity from unnecessary surgery.

There are various tracers available for LN mapping, like: vital blue dyes (MBD, IBD and Patent Blue V dye), fluorescent dyes (indocyanine green), radionuclide (Tc99m-labeled radiocolloid), Carbon nanoparticles or magnetic particles [15]. Majority of tracers require some additional equipment for imaging, like: (a) gamma camera and hand-held gamma probe or single-photon emission computed tomography for radionuclide tracers [40], (b) SPY Elite system and Hamamatsu PDE-Neo probe for indocyanine green fluorescence [41], or (c) magnetic particle imaging scanner and handheld magnetic probe if magnetic particles are used [42]. Unavailability of these tracers due to health care regulations and the costs and complexity of imaging equipment can be challenging, especially for hospitals in developing countries. Certain tracers are also associated with complications like skin tattooing, especially carbon black, reported by Zhang et al. to leave a black staining for over a year after breast surgery in $42 \%$ of patients [43]. In thyroid surgery, injection is made into the thyroid gland; however, the skin staining might occur due to tracer leakage. Anaphylactic reactions associated with vital dyes have also been reported in the literature; however, all are contributed to IBD and patent blue, none to MBD injection [44]. Indocyanine green can also lead to allergic reactions in patients with iodine or shellfish allergies, since it contains sodium iodide [41].

The uptake among tracers differs, as well as identification rate of sentinel-LNs. For example, Tc99m uptakes more rapidly than indocyanine green [41], while carbon nanoparticles that are with very small diameter have even faster uptake [43]. MBD has a fast uptake and high identification rate of sentinel-LNs, ranging from $84 \%$ in our previously published study on micro-PTCs [45] to $100 \%$ in this study on micro-MTCs. The MBD uptake can be interfered by severe thyroiditis or large goiters [45]. The accuracy of SLNB method with MBD and FSA of sentinel-LNs in reported study with micro-PTCs [45] was $97.25 \%$, while in this series of patients it reaches $100 \%$.

Given the availability, low price, feasibility of injection, speed of uptake, identification rate, accuracy and absence of anaphylactic reactions, MBD could be considered as the most appropriate tracer for SLNB in thyroid carcinomas [32, 45-47].

However, this method is not commonly used worldwide. Based on 4 meta-analyses [27, 29-31], it is mainly used for detection of central LNs metastases in differentiated thyroid carcinomas. To our knowledge, none of the SLNB studies published so far has the same surgical technique as here described [32]. Differences between original Dzodic's SLNB method, used in our center [32], and other published SLNB techniques are following: (1) cancer type: used for papillary and medullary carcinomas, (2) tracer: MBD as a single tracer, (3) neck compartment (lymphatic basin): jugulo-carotid regions, (4) pathohistological analysis: intraoperative, FSA, (5) aim of the method: immediate decision on one-time LLND. Ikeda [48] and Lee [49] also perform staging of lateral LNs; however, they use indocyanine green and Tc99m (respectively), not MBD, they do not perform FSA on sentinel-LNs, and they use it for papillary carcinomas, 
not MTC. There are only two publications on SLNB for detection of lateral LN metastases in micro-MTC after Tc99m tumor injection: one case report [50] and one pilot study [51], both published in 2014, showing that this type of LN staging is useful. However, SLNB with MBD can provide reliable results, with fewer costs and technical problems.

Although recommendations for the necessity and the extent of LLND are based on serum CT levels and preoperative US staging of LNs, there is no consensus on the most appropriate CT level-based risk stratification throughout available guidelines [1, 16-19]. A recent publication from 2019 by Norwegian authors [52] showed that basal CT cannot be used to predict the need for prophylactic LLND in patients with MTC due to many inconsistencies with CT level and presence of lateral LN metastases. In their series, lateral $\mathrm{LN}$ metastases were found in $16 \%$ of patients with $\mathrm{CT} \leq 500 \mathrm{pmol} / \mathrm{L}$ and $50 \%$ of those with CT 501-1,000 pmol/L. On the other hand, $19 \%$ of N0 patients had CT over $500 \mathrm{pmol} / \mathrm{L}$ and $17 \%$ of N1b patients had CT $\leq 500 \mathrm{pmol} / \mathrm{L}$. Similarly, out of 18 US-cN0 patients in our study that were also pathohistologically "cleared" for LN metastases (confirmed pN0), 16 of them with basal CT above $20 \mathrm{pg} / \mathrm{mL}$ were candidates for elective ipsilateral LLND, based on some recommendations [13], while 8 out of 18 with basal CT above $200 \mathrm{pg} / \mathrm{mL}$ were eligible for elective contralateral LLND. Authors strongly suggest that a benefit for patients can be observed if SLNB with MBD is performed for intraoperative LN staging and decision upon necessity for LLND, regardless the status of central LNs. On the other hand, two patients with C634F mutation were identified to harbor metastases in central and lateral compartments, although metastases were not detected by preoperative US investigation and CT values were 42.0 and $221.6 \mathrm{pg} / \mathrm{mL}$.

In our study of $20 \mathrm{cN} 0$ patients with micro-MTCs and
CT levels below $1,000 \mathrm{pg} / \mathrm{mL}$, which was expected to be a good prognostic group, we have showed that: (1) 2/20 patients actually had central and lateral LN metastases; (2) additional lateral LN metastases were found in patients who had LLND, meaning positive sentinel-LNs were predictive for metastases in other lateral LNs; and (3) patients that were "cleared" by sentinel-LN FSA to be negative did not have "skip" metastases in nonsentinel-LNs.

The authors find Dzodic's SLNB method for LN staging useful for intraoperative assessment of lateral LNs in cN0 patients and optimization of initial surgery of microMTCs with CT below 1,000 pg/mL. This way, patients with FSA proven sentinel-LN metastases can receive one-time LLND, while those with negative sentinel-LNs benefit from less extensive surgery and potentially decreased complication rate.

\section{Acknowledgements}

The authors would like to thank prof. Akira Miyauchi, Kobe, Japan, for kindly providing us necessary information on Japanese recommendations on management of medullary thyroid carcinoma, given that original guidelines are in Japanese language.

\section{Disclosure}

This research was conducted as a part of the project of the Ministry of Science of Republic of Serbia, number 111601, that started in 2005, entitled "Early detection of lymphonodal metastases by pathohistological verification of sentinel lymph nodes in malignant epithelial tumors", lead by Professor Radan Dzodic (the last author of the publication). None of the authors have any other potential conflicts of interest associated with this research.

\section{References}

1. Perros P, Boelaert K, Colley S, Evans C, Evans RM, et al. (2014) Guidelines for the management of thyroid cancer. Clin Endocrinol (Oxf) 81 Suppl 1: 1-122.

2. Scollo C, Baudin E, Travagli JP, Caillou B, Bellon N, et al. (2003) Rationale for central and bilateral lymph node dissection in sporadic and hereditary medullary thyroid cancer. J Clin Endocrinol Metab 88: 2070-2075.

3. Moley JF (2010) Medullary thyroid carcinoma: management of lymph node metastases. J Natl Compr Canc Netw 8: 549-556.

4. Baloch ZW, LiVolsi VA (2006) Microcarcinoma of the thyroid. Adv Anat Pathol 13: 69-75.

5. Beressi N, Campos JM, Beressi JP, Franc B, Niccoli-Sire
$\mathrm{P}$, et al. (1998) Sporadic medullary microcarcinoma of the thyroid: a retrospective analysis of eighty cases. Thyroid 8: 1039-1044.

6. Guyétant S, Dupre F, Bigorgne JC, Franc B, DutrieuxBerger N, et al. (1999) Medullary thyroid microcarcinoma: a clinicopathologic retrospective study of 38 patients with no prior familial disease. Hum Pathol 30: 957-963.

7. Kaserer K, Scheuba C, Neuhold N, Weinhäusel A, Haas OA, et al. (2001) Sporadic versus familial medullary thyroid microcarcinoma: a histopathologic study of 50 consecutive patients. Am J Surg Pathol 25: 1245-1251.

8. Hamy A, Pessaux P, Mirallié E, Mucci-Hennekinne S, 
Gibelin H, et al. (2005) Central neck dissection in the management of sporadic medullary thyroid microcarcinoma. Eur J Surg Oncol 31: 774-777.

9. Kazaure HS, Roman SA, Sosa JA (2012) Medullary thyroid microcarcinoma. A population-level analysis of 310 patients. Cancer 118: 620-627.

10. Machens A, Dralle H (2012) Biological relevance of medullary thyroid microcarcinoma. J Clin Endocrinol Metab 97: 1547-1553.

11. Kim JH, Pyo JS, Cho WJ (2017) Clinicopathological significance and prognosis of medullary thyroid microcarcinoma: a meta-analysis. World J Surg 41: 2551-2558.

12. Sippel RS, Kunnimalaiyaan M, Chen H (2008) Current management of medullary thyroid cancer. Oncologist 13: 539-547.

13. Machens A, Dralle H (2010) Biomarker-based risk stratification for previously untreated medullary thyroid cancer. $J$ Clin Endocrinol Metab 95: 2655-2663.

14. Turtle RM, Ball DW, Byrd D, Daniels GH, Dilawari RA, et al. (2010) Medullary Carcinoma. J Natl Compr Canc Netw 8: 512-530.

15. Kim JS (2013) Sentinel lymph node biopsy in thyroid cancer. Korean J Endocr Surg 13: 135-143.

16. Dralle H, Musholt TJ, Schabram J, Steinmüller T, Frilling A, et al. (2013) German Association of Endocrine Surgeons practice guideline for the surgical management of malignant thyroid tumors. Langenbecks Arch Surg 398: 347-375.

17. Wells SA Jr, Asa SL, Dralle H, Elisei R, Evans DB, et al. (2015) Revised American Thyroid Association guidelines for the management of medullary thyroid carcinoma. Thyroid 25: 567-610.

18. Mitchell AL, Gandhi A, Scott-Coombes D, Perros P (2016) Management of thyroid cancer: United Kingdom National Multidisciplinary Guidelines. J Laryngol Otol 130: S150-S160.

19. The Japanese Society of Thyroid Surgery/The Japan Association of Endocrine Surgeons (2018) Guidelines for the Management of Thyroid Tumors. Journal of the Japan Association of Endocrine Surgeons and the Japanese Society of Thyroid Surgery 35 Suppl 3: G1-G68 (In Japanese).

20. Russell CF, Van Heerden JA, Sizemore GW, Edis AJ, Taylor WF, et al. (1983) The surgical management of medullary thyroid carcinoma. Ann Surg 197: 42-48.

21. Shaha AR (1998) Management of the neck in thyroid cancer. Otolaryngol Clin North Am 31: 823-831.

22. Kebebew E, Clark OH (2000) Medullary thyroid cancer. Curr Treat Options Oncol 1: 359-367.

23. Machens A, Gimm O, Ukkat J, Hinze R, Schneyer U, et al. (2000) Improved prediction of calcitonin normalization in medullary thyroid carcinoma patients by quantitative lymph node analysis. Cancer 88: 1909-1915.

24. Kelemen PR, Van Herle AJ, Giuliano AE (1998) Sentinel lymphadenectomy in thyroid malignant neoplasms. Arch Surg 133: 288-292.

25. Roh JL, Park CI (2008) Sentinel lymph node biopsy as guidance for central neck dissection in patients with papil- lary thyroid carcinoma. Cancer 113: 1527-1531.

26. Anand SM, Gologan O, Rochon L, Tamilia M, How J, et al. (2009) The role of sentinel lymph node biopsy in differentiated thyroid carcinoma. Arch Otolaryng Head Neck Surg 135: 1199-1204.

27. Raijmakers PG, Paul MA, Lips P (2008) Sentinel node detection in patients with thyroid carcinoma: a metaanalysis. World J Surg 32: 1961-1967.

28. Cunningham DK, Yao KA, Turner RR, Singer FR, Van Herle AR, et al. (2010) Sentinel lymph node biopsy for papillary thyroid cancer: 12 years of experience at a single institution. Ann Surg Oncol 17: 2970-2975.

29. Balasubramanian SP, Harrison BJ (2011) Systematic review and meta-analysis of sentinel node biopsy in thyroid cancer. Br J Surg 98: 334-344.

30. Kaczka K, Celnik A, Luks B, Jasion J, Pomorski L (2012) Sentinel lymph node biopsy techniques in thyroid pathologies - a meta-analysis. Endokrynol Pol 63: 222-231.

31. Garau LM, Rubello D, Ferretti A, Boni G, Volterrani D, et al. (2018) Sentinel lymph node biopsy in small papillary thyroid cancer. A review on novel surgical techniques. Endocrine 62: 340-350.

32. Dzodic R, Markovic I, Inic M, Jokic N, Djurisic I, et al. (2006) Sentinel lymph node biopsy may be used to support the decision to perform modified radical neck dissection in differentiated thyroid carcinoma. World J Surg 30: 841-846. Erratum in: World J Surg 2006; 30: 918.

33. Dzodic R, Santrac N (2017) In situ preservation of parathyroid glands: advanced surgical tips for prevention of permanent hypoparathyroidism in thyroid surgery. $J$ BUON 22: 853-855.

34. Wells SA, Gunnells JC, Shelburne JD, Schneider AB, Sherwood LM (1975) Transplantation of the parathyroid glands in man: clinical indications and results. Surgery 78: 34-44.

35. Karges W, Dralle H, Raue F, Mann K, Reiners C, et al. (2004) Calcitonin measurement to detect medullary thyroid carcinoma in nodular goiter: German evidence-based consensus recommendations. Exp Clin Endocrinol Diabetes 112: 52-58.

36. Moley JF, DeBenedetti MK (1999) Patterns of nodal metastases in palpable medullary thyroid carcinoma: recommendations for extent of node dissection. Ann Surg 229: 880-888.

37. Dralle H, Damm I, Scheumann GF, Kotzerke J, Kupsch E, et al. (1994) Compartment-oriented microdissection of regional lymph nodes in medullary thyroid carcinoma. Surg Today 24: 112-121.

38. Machens A, Hauptmann S, Dralle H (2008) Prediction of lateral lymph node metastases in medullary thyroid cancer. Br J Surg 95: 586-591.

39. Moley JF, Dilley WG, DeBenedetti MK (1997) Improved results of cervical reoperation for medullary thyroid carcinoma. Ann Surg 225: 734-740.

40. Garcia-Burillo A, Roca Bielsa I, Gonzalez O, Zafon C, Sabate M, et al. (2013) SPECT/CT sentinel lymph node identification in papillary thyroid cancer: lymphatic stag- 
ing and surgical management improvement. Eur $\mathrm{J} \mathrm{Nucl}$ Med Mol Imaging 40: 1645-1655.

41. McGregor A, Pavri SN, Tsay C, Kim S, Narayan D (2017) Use of indocyanine green for sentinel lymph node biopsy: case series and methods comparison. Plast Reconstr Surg Glob Open 5: e1566.

42. Gräfe K, Sattel TF, Lüdtke-Buzug K, Finas D, Borgert J, et al. (2012) Magnetic-particle-imaging for sentinel lymph node biopsy in breast cancer. In: Buzug T, Borgert J (eds) Magnetic Particle Imaging. Springer Proceedings in Physics, vol. 140. Springer, Berlin, Heidelberg: 237-241.

43. Zhang L, Huang Y, Yang C, Zhu T, Lin Y, et al. (2018) Application of a carbon nanoparticle suspension for sentinel lymph node mapping in patients with early breast cancer: a retrospective cohort study. World J Surg Oncol 16: 112.

44. Bézu C, Coutant C, Salengro A, Daraï E, Rouzier R, et al. (2011) Anaphylactic response to blue dye during sentinel lymph node biopsy. Surg Oncol 20: e55-e59.

45. Goran M, Pekmezovic T, Markovic I, Santrac N, Buta M, et al. (2017) Lymph node metastases in clinically N0 patients with papillary thyroid microcarcinomas - a single institution experience. $J$ BUON 22: 224-231.

46. Santrac N, Besic N, Buta M, Oruci M, Djurisic I, et al. (2014) Lymphatic drainage, regional metastases and surgical management of papillary thyroid carcinoma arising in pyramidal lobe - a single institution experience. Endocr $J$
61: 55-59.

47. Dzodic R, Buta M, Markovic I, Gavrilovic D, Matovic M, et al. (2014) Surgical management of well-differentiated thyroid carcinoma in children and adolescents: 33 years of experience of a single institution in Serbia. Endocr $J$ 61: 1079-1086.

48. Ikeda Y (2011) Sentinel lymph node biopsy as guidance for lateral neck dissection in patients with papillary thyroid carcinoma. Surg Sci 2: 57-61.

49. Lee SK, Kim SH, Hur SM, Choe JH, Kim JH, et al. (2011) The efficacy of lateral neck sentinel lymph node biopsy in papillary thyroid carcinoma. World J Surg 35: 2675-2682.

50. Boni G, Mazzarri S, Grosso M, Manca G, Biricotti M, et al. (2014) Sentinel node radioguided biopsy in surgical management of the medullary thyroid carcinoma-A case report. Ann Ital Chir 85 (ePub).

51. Puccini M, Manca G, Ugolini C, Candalise V, Passaretti A, et al. (2014) Interest of sentinel node biopsy in apparently intrathyroidal medullary thyroid cancer: a pilot study. J Endocrinol Invest 37: 829-834.

52. Opsahl EM, Akslen LA, Schlichting E, Aas T, Brauckhoff $\mathrm{K}$, et al. (2019) The role of calcitonin in predicting the extent of surgery in medullary thyroid carcinoma: a nationwide population-based study in Norway. Eur Thyroid $J$ 8: 159-166. 